Faculty \& Staff Scholarship

2006

Hybrid Ant Systems for the Dynamic Facility Layout Problem

Alan McKendall

Jin Shang

Follow this and additional works at: https://researchrepository.wvu.edu/faculty_publications

Part of the Operations Research, Systems Engineering and Industrial Engineering Commons 


\title{
Hybrid ant systems for the dynamic facility layout problem
}

\author{
Alan R. McKendall Jr.*, Jin Shang \\ Department of Industrial and Management Systems Engineering, West Virginia University, 325A Mineral Resources Building, \\ Morgantown, WV 26506, USA
}

Available online 22 September 2004

\begin{abstract}
Today's consumer market demands that manufacturers must be competitive. This requires the efficient operation of manufacturing plants and their ability to quickly respond to changes in product mix and demand. In addition, studies show that material-handling cost make up between 20 and 50 percent of the total operating cost. Therefore, this paper considers the problem of arranging and rearranging, when there are changes in product mix and demand, manufacturing facilities such that the sum of material handling and rearrangement costs is minimized. This problem is called the dynamic facility layout problem (DFLP). In this paper, hybrid ant systems (HASs) are developed to solve the DFLP. To test the performance of the meta-heuristics, two data sets taken from the literature are used in the analysis. The results show that the HASs are efficient techniques for solving the DFLP. More importantly, HASs found new best solutions for more than one-half of all the test problems.

(C) 2004 Elsevier Ltd. All rights reserved.
\end{abstract}

Keywords: Dynamic facility layout problem; Hybrid ant system; Ant colonies; Simulated annealing; Heuristics

\section{Introduction}

The facility layout problem is the arrangement of departments within a facility with respect to some objective. Since studies show that material-handling cost makes up between 20 and 50 percent of the total operating cost and between 15 and 70 percent of the total cost of manufacturing a product (Tompkins et al. [1, p. 138]), the most common objective considered is the minimization of material-handling cost. Material-handling costs are determined based on the amounts of materials that flow between the departments and the distances between the locations of the departments. When the flow of materials

\footnotetext{
* Corresponding author. Fax: +1-304-293-4970.

E-mail address: armckendall@mail.wvu.edu (A.R. McKendall Jr.).
} 
between the departments is fixed during the planning horizon, this problem is known as the static (single period) facility layout problem (SFLP), which can be formulated as a quadratic assignment problem (QAP). For a review of the SFLP literature, see Meller and Gau [2]. When the flow of materials between departments changes during the planning horizon, this problem is known as the dynamic (multipleperiod) facility layout problem (DFLP). Some of the factors associated with changes in the flow between departments are as follows and were taken from Shore and Tompkins [3].

- Changes in the design of an existing product;

- the addition or deletion of a product;

- replacement of existing production equipment;

- shorter product life cycles and

- changes in the production quantities and associated production schedule.

For the DFLP, it is assumed that the flow data for each period remains constant throughout the period. Therefore, the layout for each period in the planning horizon can be obtained by solving the SFLP for each period using the QAP formulation. If $\pi_{t}$ is used to represent the layout for each time period $t(t=1,2, \ldots, T)$ with $N$ departments, then one solution representation is $\pi_{t}=\left(\pi_{1 t}, \pi_{2 t}, \ldots, \pi_{N t}\right)$, where $\pi_{i t}$ represents the department assigned to location $i(i=1,2, \ldots, N)$ at time period $t$. Hence, a solution representation for the DFLP is

$$
\pi=\left\{\pi_{1}, \pi_{2}, \ldots, \pi_{T}\right\}=\left\{\left(\pi_{11}, \pi_{21}, \ldots, \pi_{N 1}\right),\left(\pi_{12}, \pi_{22}, \ldots, \pi_{N 2}\right), \ldots,\left(\pi_{1 T}, \pi_{2 T}, \ldots, \pi_{N T}\right)\right\} .
$$

The material-handling cost for each layout $\pi_{t}$ in each time period $t$ can be obtained by calculating

$$
\sum_{i=1}^{N} \sum_{j=1}^{N} f_{\pi_{i t} \pi_{j t}} d_{i j} \quad \text { for } t=1,2, \ldots, T,
$$

where $f_{\pi_{i t} \pi_{j t}}$ and $d_{i j}$ represent the flow of materials between departments $\pi_{i t}$ and $\pi_{j t}$ in time period $t$ and the distance between locations $i$ and $j$, respectively. As a result, the total material-handling cost for the layout plan, $\pi$, is

$$
\sum_{i=1}^{N} \sum_{j=1}^{N} \sum_{t=1}^{T} f_{\pi_{i t} \pi_{j t}} d_{i j}
$$

If the layout between consecutive periods changes (i.e., the locations of two or more departments change), then the cost of moving departments from one location to another needs to be considered. This cost is called rearrangement cost. The rearrangement cost is

$$
\sum_{i=1}^{N} \sum_{t=2}^{T} r_{\pi_{i t}} x_{i t}
$$

where $r_{\pi_{i t}}$ is the arrangement cost for moving department $\pi_{i t}$ to location $i$ in period $t$. The variable $x_{i t}$ ensures that if department $\pi_{i t}$ assigned to location $i$ at period $t$ is different from the department $\pi_{i(t-1)}$ assigned to location $i$ at period $t-1$, then rearrangement cost is incurred. This is considered by 


\begin{tabular}{|l|l|l|}
\hline 3 & 5 & 1 \\
\hline 2 & 4 & 6 \\
\hline
\end{tabular}

$t=1$

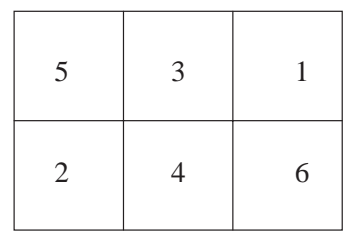

$t=2$

\begin{tabular}{|l|l|l|}
\hline 5 & 3 & 1 \\
\hline 2 & 4 & 6 \\
\hline
\end{tabular}

$t=3$

Fig. 1. A DFLP instance with 6 departments and 3 time periods.

defining

$$
x_{i t}= \begin{cases}1 & \text { if } \pi_{i t} \neq \pi_{i(t-1)} \\ 0 & \text { otherwise. }\end{cases}
$$

See Fig. 1 for a DFLP instance with 6 departments and 3 time periods in the planning horizon. In period 1, departments $3,5,1,2,4$, and 6 are assigned to locations $1,2,3,4,5$, and 6 , respectively. The layout of period 1 is represented as the permutation $\pi_{1}=(3,5,1,2,4,6)$, where departments 3 and 5 are assigned to locations 1 and 2 (i.e., $\pi_{11}=3$ and $\pi_{21}=5$ ), respectively, and so on. The layout plan for the DFLP instance is

$$
\pi=\{(3,5,1,2,4,6),(5,3,1,2,4,6),(5,3,1,2,4,6)\} .
$$

Since departments 3 and 5 are assigned to different locations (i.e., locations 2 and 1, respectively) in period 2 , rearrangement costs for period 2 is the cost of moving department 3 to location 2 plus the cost of moving department 5 to location 1. Because the layout is the same in periods 2 and 3, there are no rearrangement costs in period 3. Also, the material-handling costs for each period can be determined. Therefore, the DFLP is the problem of efficiently arranging the departments within a facility during a multi-period planning horizon such that the sum of material handling and rearrangement costs is minimized.

Rosenblatt [4] presented a dynamic programming algorithm to solve the DFLP optimally. However, since the number of possible solutions or layout plans is $(N !)^{T}$ for DFLP instances with $N$ departments and $T$ time periods, only small problem instances can be solved optimally in reasonable time. Therefore, the author presented two heuristics using dynamic programming. The approach is similar to the approach presented by Ballou [5] for a dynamic warehouse location problem. Lacksonen and Enscore [6] modified five heuristics to solve the DFLP: a heuristic based on the dynamic programming algorithm presented in Rosenblatt [4]; a heuristic based on a branch and bound algorithm for the QAP presented by Pardalos and Crouse [7]; a heuristic which is a modification of the cutting plane algorithm for the QAP developed by Burkard and Bonniger [8]; a heuristic based on cut trees presented by Gomory and Hu [9]; and a heuristic similar to CRAFT (Armour and Buffa [10]) for the QAP. Urban [11] developed a steepest descent pairwise exchange technique similar to CRAFT presented by Armour and Buffa [10], which consider forecast windows. Conway and Venkataramanan [12] used a genetic algorithm to solve the DFLP, and Kaku and Mazzola [13] used a tabu search heuristic. Balakrishnan and Cheng [14] improved the genetic algorithm presented in Conway and Venkataramanan [12]. Also, Balakrishnan et al. [15] improved the pairwise exchange heuristic with time windows presented in Urban [11] by presenting a backward-pass pairwise exchange heuristic with forecast windows. The authors also presented a heuristic, which combines dynamic programming and Urban's pairwise exchange heuristic with time windows. 
Baykasoglu and Gindy [16] presented a simulated annealing (SA) heuristic, and Balakrishnan et al. [17] presented a hybrid genetic algorithm for the DFLP. For a review of the problem assumptions and solution approaches for the DFLP, see Balakrishnan and Cheng [18].

More recently, new techniques, which use the idea of the way ants forage for food and find the shortest paths between a food source and the colony, have been used to solve combinatorial optimization problems such as the traveling salesperson problem (Dorigo and Gambardella [19]) and the QAP (Gambardella et al. [20]). The shortest path between a food source and the colony is determined based on the chemical trail (called the pheromone trail) an ant leaves while returning back to the colony from the food source so that other ants can find the food source. The amount of the pheromone trail left by an ant is based on the amount of food found. If the paths between the food source and the colony are far apart, fewer ants will travel these paths, and the trail will eventually evaporate. However, when the path between the food source and the colony are close, more ants will travel this path, which will be indicated by a strong pheromone trail.

In this paper, hybrid ant systems (HASs) are developed for the DFLP, which are modifications of the hybrid ant colony system applied to the QAP (HAS-QAP) presented by Gambardella et al. [20]. In Section 2, the descriptions of the HASs are presented for the DFLP. The computational results of the HASs on two sets of test problems taken from the literature are given in Section 3 followed by conclusions, in Section 4 .

\section{HASs for the DFLP}

In this section, HASs for the DFLP are presented. Ant colonies were first proposed by Colorni et al. [21] as a solution technique for solving the traveling salesman problem. More often, these algorithms are construction algorithms used to generate solutions from scratch. However, several authors developed hybrid ant algorithms for the QAP such as Gambardella et al. [20], Maniezzo and Colorni [22], and Stutzle and Dorigo [23]. Among them, Gambardella et al. [20] proposed the so-called HAS (HAS-QAP). This technique was modified for the DFLP and is briefly summarized below. This technique is called HAS I.

The HAS I heuristic starts with an initial set of layout plans (i.e., $M$ solutions or ants). The initial set of $M$ solutions is improved using a pairwise exchange heuristic, and the cost of each layout plan is obtained. The minimum cost is used to initialize the pheromone trail matrix $P$. The entries of matrix $P$, $p_{\pi_{i t} j t}$, measures the desirability of assigning department $\pi_{i t}$ in location $i$ to location $j$ at time period $t$. At the initial or current iteration, each layout plan is modified using the pheromone trail matrix. More specifically, a layout plan is modified by performing department location exchanges using the pheromone trail matrix and one of two probabilistic policies, which will be explained later. The modified solutions are improved using a random descent pairwise exchange heuristic. If necessary, the best solution is updated. Next, an intensification strategy is used to determine the set of starting solutions for the next iteration (i.e., a solution for each ant), and the pheromone trails (entries in matrix $P$ ) are updated such that all the trails are first weakened and then, the pheromone trails corresponding to the best solution are strengthened. Also, a diversification strategy is implemented. In other words, after $S$ iterations without improving the best solution, the solutions (all except the best solution) and the pheromone trail matrix are erased, and the heuristic is started from the beginning where only the best solution (or ant) and the current iteration number are used. As a result, the other $M-1$ initial solutions are randomly generated. 
A pairwise exchange technique is used in the HAS I heuristic to improve the initial set of solutions and the set of modified solutions, obtained using the pheromone trail matrix. Hence, the pairwise exchange heuristic is an important component of the HAS I heuristic. The steps for the pairwise exchange heuristic for the HAS I heuristic are as follows.

Step 1: Given a layout plan (or solution) $\pi=\left\{\pi_{1}, \pi_{2}, \ldots, \pi_{T}\right\}$ and its cost $f(\pi)$. Randomly select a time period $t(t=1,2, \ldots, T)$.

Step 2: Randomly select two departments, $\pi_{i t}$ and $\pi_{j t}$, for exchange in period $t$, resulting in a new layout plan $\pi^{\prime}$.

Step 3: Calculate the change in total cost, $\Delta f=f(\pi)-f\left(\pi^{\prime}\right)$. If $\Delta f>0$, accept the exchange, and set $\pi=\pi^{\prime}$. Otherwise, reject the exchange.

This pairwise exchange technique is a random descent heuristic, which is repeated $N * N * T$ times for each ant. The change in total cost $(\Delta f)$ is the sum of the change in material-handling cost and in rearrangement cost, as a result of exchanging the locations of departments $\pi_{i t}$ and $\pi_{j t}$ in period $t$.

The following is the HAS I heuristic developed for the DFLP, which is a modification of the HAS-QAP heuristic presented by Gambardella et al. [20].

Step 1: Randomly generate a set of initial solutions or layout plans $\pi^{1}, \pi^{2}, \ldots, \pi^{M}$, which represents a colony of $M$ ants, and calculate the costs of each layout plan (i.e., $\left.f\left(\pi^{1}\right), f\left(\pi^{2}\right), \ldots, f\left(\pi^{M}\right)\right)$.

Step 2: Use the pairwise exchange heuristic to improve the solution or layout plan for each ant, and let $\pi^{*}$ represent the best solution or ant such that $f\left(\pi^{*}\right)=\min \left\{f\left(\pi^{1}\right), f\left(\pi^{2}\right), \ldots, f\left(\pi^{M}\right)\right\}$.

Step 3: Set the heuristic parameters:

$Q=$ parameter used to determine the initial values of the pheromone trail matrix $P$.

$R=$ number of exchanges used to modify each layout plan.

$q=$ probability that policy 1 will be selected to modify a layout plan. As a result, policy 2 is chosen with a probability of $1-q$.

$\alpha_{1}, \alpha_{2}=$ parameters used to weaken and enforce pheromone trails, respectively.

$S=$ number of consecutive iterations, without improving best solution $\pi^{*}$, before implementing diversification strategy.

Step 4: Initialize the pheromone trail matrix $P$.

Step 5: Modify the solutions $\pi^{1}, \pi^{2}, \ldots, \pi^{M}$ using the pheromone trail matrix and one of two policies, which is discussed below. The modified solutions are represented as $\tilde{\pi}^{1}, \tilde{\pi}^{2}, \ldots, \tilde{\pi}^{M}$.

Step 6: Use the pairwise exchange heuristic to improve the solutions $\tilde{\pi}^{1}, \tilde{\pi}^{2}, \ldots, \tilde{\pi}^{M}$ and obtain $\hat{\pi}^{1}, \hat{\pi}^{2}, \ldots, \hat{\pi}^{M}$. Update the best solution $\pi^{*}$ and $f\left(\pi^{*}\right)$.

Step 7: If the best solution $\pi^{*}$ has not improved after $S$ consecutive iterations, then perform the diversification strategy and go to Step 4. Otherwise, use the intensification strategy (discussed below) to determine the set of solutions $\pi^{1}, \pi^{2}, \ldots, \pi^{M}$, which will be used to start the next iteration.

Step 8: Update the pheromone trail matrix $P$, and go to Step 5.

First, a permutation (or layout) for the first period is generated by assigning departments $1,2, \ldots, N$ to locations $1,2, \ldots, N$, respectively. Then this layout is used for all of the remaining periods (i.e., $t=2,3, \ldots, T)$, and all the ant are assigned this layout plan. Thus, the layout plans for all ants are the same. Therefore, the rearrangement cost is zero. Next, calculate the total material-handling cost, which gives the total cost of the layout plan. In Step 2, the pairwise exchange heuristic is used to improve the initial solution so that the best solution $\pi^{*}$ can be used to initialize the pheromone trail matrix in Step 4. Entries of matrix $P, p_{\pi_{i t} j}$, represents the desirability of assigning department $\pi_{i t}$ in location $i$ to location $j$ at time period $t$. In Step 4, all the entries of $P$ are set to $Q / f\left(\pi^{*}\right)$, where $Q$ is a heuristic parameter 
used to control the initial values of $P$. In Step 5, each of the solutions $\pi^{1}, \pi^{2}, \ldots, \pi^{M}$ are modified by performing $R$ department location exchanges, using the pheromone trail matrix. First, a period $t$ and a department $\pi_{u t}$ are randomly selected. Second, a location $v \neq u$ is selected using one of the following two policies:

(1) Location $v$ is chosen such that $p_{\pi_{u t} v t}+p_{\pi_{v t} u t}$ is maximized.

(2) Location $v$ is chosen with probability $\frac{p_{\pi_{u t} v t}+p_{\pi_{v t} u t}}{\sum_{j \neq u}\left(p_{\pi_{u t} j t}+p_{\pi_{j t} u t}\right)}$.

The first policy is selected with a probability of $q$. This policy uses the pheromone trail to select a location $v$ based on the most desirable exchange between the department assigned to location $u$ and each department assigned to all locations except location $u$ (ties are broken randomly). The second policy selects a location $v$ randomly, but there is a higher probability that the $v$ with the largest $p_{\pi_{u t} v t}+p_{\pi_{v t} u t}$ will be accepted. After selecting a location $v$, the departments assigned to locations $u$ and $v$ are exchanged. This procedure is executed $R$ times for each ant, and the modified solutions $\tilde{\pi}^{1}, \tilde{\pi}^{2}, \ldots, \tilde{\pi}^{M}$ are obtained. In Step 6 , the solutions $\tilde{\pi}^{1}, \tilde{\pi}^{2}, \ldots, \tilde{\pi}^{M}$ are improved by using the pairwise exchange heuristic, and the solutions $\hat{\pi}^{1}, \hat{\pi}^{2}, \ldots, \hat{\pi}^{M}$ are obtained. Also, the best solution $\pi^{*}$ and $f\left(\pi^{*}\right)$ are updated, if they have changed.

In Step 7, if the best solution $\pi^{*}$ has not improved after $S$ consecutive iterations, a diversification strategy is implemented. That is, the best solution $\pi^{*}$ and a new set of $M-1$ layout plans or solutions $\pi^{1}, \pi^{2}, \ldots, \pi^{M-1}$, which are randomly generated as defined in Step 1, are used to start the next iteration. The $M-1$ solutions are improved by using the pairwise exchange heuristic, and the best solution $\pi^{*}$ is obtained out of the $M$ solutions and used to re-initialize the pheromone trail matrix $P$, as defined in Steps 2 and 4, respectively. Then, the heuristic continues, as before at Step 5.

If the diversification strategy is not implemented, then the intensification strategy may be performed. Initially, the intensification strategy is activated, which means that the better solution between $\pi^{k}$ and $\hat{\pi}^{k}$ for each ant $k$ is used as the starting solution for the next iteration. This strategy remains active if at least one ant improves its solution during an iteration. Otherwise, the intensification strategy is inactive, and each ant $k$ starts its next iteration with the solution $\hat{\pi}^{k}$. If the strategy is inactive, it becomes activated when the best solution $\pi^{*}$ is improved.

After obtaining the starting solutions for the next iteration for each ant (i.e., $\pi^{1}, \pi^{2}, \ldots, \pi^{M}$ ), update the pheromone trail matrix $P$, in Step 8. First, all of the trails are weakened by using the formula

$$
p_{\pi_{i t} j t}=\left(1-\alpha_{1}\right) p_{\pi_{i t} j t}
$$

where $0<\alpha_{1}<1$ is a parameter used to control the evaporation of the trails. A value close to 0 indicates that the trails will remain active for a long time, and a value close to 1 indicates a high degree of evaporation and a shorter memory of the system. Afterwards, pheromone trails corresponding to the best solution $\pi^{*}$ are reinforced by using the formula

$$
p_{\pi_{i t}^{*} i t}=p_{\pi_{i t}^{*} i t}+\alpha_{2} / f\left(\pi^{*}\right)
$$

where $\alpha_{2}$ is a parameter used to control the reinforcement of the pheromone trails corresponding to the best solution $\pi^{*}$. Using only the best solution $\pi^{*}$ speeds up the convergence of the heuristic (Gambardella et al. [20]). Once the pheromone trail matrix $P$ is updated, the next iteration continues at Step 5. The heuristic is terminated after running for a pre-defined number of minutes. 
The second heuristic, called the HAS II heuristic, uses the ideas of the HAS I and SA heuristics. SA is a stochastic approach for solving combinatorial optimization problems, in which the basic idea comes from the annealing process of solids. In this process, a solid is heated until it melts, and then the temperature of the solid is slowly decreased (according to an annealing schedule) until the solid reaches the lowest energy state (or ground state). If the initial temperature is not high enough or if the temperature is decreased rapidly, the solid at the ground state will have many defects or imperfections. Kirkpatrick et al. [24] were the first to use SA to solve combinatorial optimization problems. As well as others, Wilhelm and Ward [25] and Heragu and Alfa [26] presented SA heuristics for the SFLP. As mentioned previously, Baykasoglu and Gindy [16] presented the first SA heuristic for the DFLP.

The HAS II heuristic performs Steps 1-8 of the HAS I heuristic. However, a SA heuristic is used in place of the pairwise exchange heuristic in Steps 2 and 6. The steps for the SA heuristic for the HAS II heuristic are as follows.

Step 0: Define the SA parameters: $T_{0}=$ initial temperature, $\alpha=$ cooling ratio, $A M=2(N)\left(T^{2}\right)=$ attempted number of moves at each temperature, $T_{-} \min =0.01=$ minimum temperature.

Step 1: Initialize the temperature change counter: $r=1$.

Step 2: Given a layout plan (or solution) $\pi=\left\{\pi_{1}, \pi_{2}, \ldots, \pi_{T}\right\}$ and its cost $f(\pi)$. Set current solution and Best_sol $=\pi$ and Best_cost $=f(\pi)$.

Step 3: If current temperature $T \leqslant T_{-}$min, then stop and return Best_sol $=\pi$ and Best_cost $=f(\pi)$. Else, initialize counter for the number of attempted moves at each temperature: $a=0$, and set the current temperature according to the annealing schedule, $T=T_{0} \alpha^{r-1}$.

Step 4: Randomly select a time period $t(t=1,2, \ldots, T)$, and randomly select two departments, $\pi_{i t}$ and $\pi_{j t}$, for exchange in period $t$ such that $i \neq j$, resulting in a new layout plan $\pi^{\prime}$.

Step 5: Update $a=a+1$, and calculate the change in total cost, $\Delta f=f\left(\pi^{\prime}\right)-f(\pi)$.

If $(\Delta f<0)$ or $(\Delta f>0$ and $x=\operatorname{random}(0,1)<P(\Delta f)=\exp (-\Delta f / T))$, accept the exchange, (i.e., set $\left.\pi=\pi^{\prime}\right)$, and

if Best_cost $>f(\pi)$, then set Best_cost $=f(\pi)$, and Best_sol $=\pi$. Otherwise, reject the exchange.

Step 6: If $a \geqslant A M$, then update $r=r+1$, and go to Step 3. Else, go to Step 4 .

This SA technique is repeated for each ant. In order to determine the parameter settings for the SA heuristic, both theoretical and experimental techniques were used. The initial temperature $T_{0}$ was determined by using the formula $P(\Delta f)=\exp \left(-\Delta f / T_{0}\right)$ such that $\Delta f=0.10 f(\pi), P(\Delta f)=0.25$, where 0.10 and 0.25 were obtained experimentally. In other words, the initial temperature is obtained by the formula $T_{0}=-0.10 f(\pi) / \ln (0.25)$, which means that there is a $25 \%$ chance of accepting a solution $10 \%$ more than the cost of $\pi$. Furthermore, $\alpha$ and $A M$ were set to 0.99 and $2(N)\left(T^{2}\right)$, which were obtained experimentally.

The third heuristic, called the HAS III heuristic, adds a look-ahead/look-back strategy to the pairwise exchange heuristic within the HAS I heuristic. Similarly, the pairwise exchange heuristics for HAS I and III randomly selects a period $t$ and the locations $i$ and $j$ of two departments for exchange, and the change in the total cost $\Delta(f)$ is obtained. If $\Delta f>0$, then the exchange is accepted. Otherwise, it is rejected, and the heuristic starts from the beginning. If the exchange is accepted in the simple pairwise exchange heuristic in HAS I, then the exchange is performed, and the current solution is updated. This process is repeated for $N * N * T$ iterations. However, if the exchange is accepted in the pairwise exchange heuristic in HAS III, then the exchange is performed, and the current solution is updated. In addition, the changes in the total cost of performing the exchange in the preceding $(t-1)$ and succeeding $(t+1)$ periods 
are considered. If the exchange is accepted for both periods, update the current solution and consider the exchange in the preceding and succeeding $(t-2$ and $t+2)$ periods. If the exchange is accepted for only one of the periods, say $t-1$, then update the current solution, consider the exchange in the previous $(t-2)$ period, and reject the exchange in period $t+1$. Continue considering the exchange in the preceding or succeeding periods until there are no more periods to consider or the exchange does not improve the solution (i.e., $\Delta f \leqslant 0$ ). If the exchange is rejected for both periods, then repeat the above process for $N * N * T$ iterations. The diagram of the look-ahead/look-back pairwise exchange heuristic is given in Fig. 2.

\section{Computational results}

In this section, the computational results of the HASs applied to 80 DFLP instances are presented. The first 32 test problems (data set 1), which were provided by Lacksonen and Enscore [6], contain problems with $6,12,20$, and 30 departments each with 3 and 5 time periods. The remaining 48 test problems (data set 2), which were obtained from Balakrishnan and Cheng [14], contain problems with 6, 15, and 30 departments each with 5 and 10 time periods. The proposed HAS heuristics were programmed using the $\mathrm{C}++$ programming language, and the two sets of test problems were solved on a Pentium IV $2.4 \mathrm{GHz}$ PC. Each test problem was solved three times for each heuristic.

All the parameter settings for the proposed HAS heuristics were obtained experimentally and are given in Tables 1 and 2 for data sets 1 and 2, respectively. Each test problem was solved three times for each heuristic, and the best solution was recorded. Tables 3 and 4 summarize the results obtained for data sets 1 and 2, respectively, in the HAS I, HAS II, and HAS III columns. The bold numbers gives the best solution for each test problem. The best solution found in the literature and the best solution found by a HAS heuristic are given in columns 6 and 10, respectively. Also, the percent deviations of the best HAS solutions away from the best found solutions are given in the last column. For data set 1, the results for the HAS heuristics are compared to the results obtained by the tabu search (TS) heuristics presented by Kaku and Mazzola [13] and the cutting planes (CP) algorithm presented by Lacksonen and Enscore [6]. For data set 2, the HAS results are compared to the best results obtained by the SA heuristic presented by Baykosaglu and Gindy [16] and the hybrid genetic algorithm (GADP) presented by Balakrishnan et al. [17]. It is important to note that the correct results for the SA heuristic presented by Baykosaglu and Gindy [16] were taken from Baykosaglu and Gindy [27]. The HAS heuristics are compared to the above heuristics since these heuristics are considered to be the best heuristics in the literature for the DFLP.

In Table 3, the solutions with an asterisk are the solutions that Lacksonen and Enscore [6] verified as the optimal. Therefore, the CP, TS, HAS I, HAS II, and HAS III heuristics obtained the optimal solutions for 7 out of the 8 six-department test problems, and all except $\mathrm{CP}$ obtained the best solution for problem 6. For the 12-, 20- and 30-department problems, the HAS I heuristic produced the best solutions for 8, 5 , and 1 of the test problems, respectively. The HAS II heuristic produced the best solution for 8,6 , and 3 of the test problems, respectively, and the HAS III heuristic produced the best solution for 8, 7, and 8 of the test problems, respectively. In other words, out of the 24 problems (i.e., the problems with 12, 20, and 30 departments), the HAS I, II, and III obtained the best solution for 14, 17, and 23 of the problems, respectively. Therefore, HAS III clearly outperformed HAS I and II, and HAS II outperformed HAS I for this data set. For the 32 test problems, the CP and TS heuristics obtained the best solution for 18 and 25 problems, respectively. However, the HAS I, II, and III obtained the best solution for 22, 25 , 


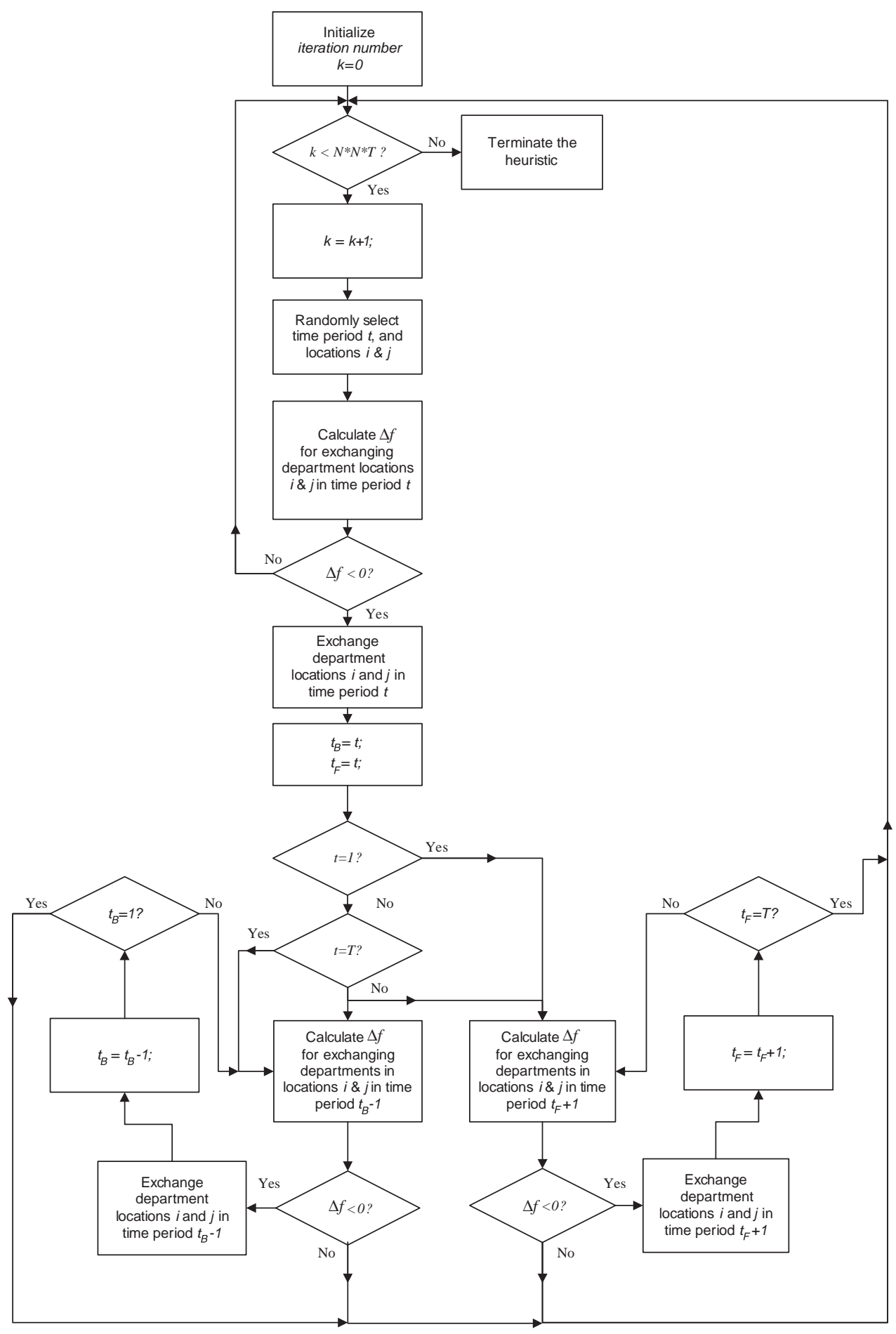

Fig. 2. Diagram of the look-ahead/look-back pairwise exchange procedure. 
Table 1

HAS parameter settings for data set 1

\begin{tabular}{|c|c|c|c|c|c|c|c|c|c|}
\hline $\begin{array}{l}\text { No. of } \\
\text { departments }\end{array}$ & $\begin{array}{l}\text { No. of time } \\
\text { periods }\end{array}$ & $M$ & $Q$ & $q$ & $\alpha_{1}$ & $\alpha_{2}$ & $S$ & $R$ & $\begin{array}{l}\text { Running time } \\
\text { (min) }\end{array}$ \\
\hline \multirow[t]{2}{*}{6} & 3 & 5 & $2 \times 10^{4}$ & 0.9 & 0.1 & $2 \times 10^{4}$ & 100 & 3 & 0.1 \\
\hline & 5 & 5 & $2 \times 10^{4}$ & 0.9 & 0.1 & $2 \times 10^{4}$ & 150 & 3 & 0.5 \\
\hline \multirow[t]{2}{*}{12} & 3 & 10 & $5 \times 10^{4}$ & 0.9 & 0.1 & $5 \times 10^{4}$ & 200 & 3 & 1.5 \\
\hline & 5 & 10 & $5 \times 10^{4}$ & 0.9 & 0.1 & $5 \times 10^{4}$ & 250 & 3 & 3.0 \\
\hline \multirow[t]{2}{*}{20} & 3 & 15 & $1 \times 10^{4}$ & 0.8 & 0.2 & $1 \times 10^{4}$ & 300 & 2 & 4.5 \\
\hline & 5 & 20 & $1 \times 10^{4}$ & 0.8 & 0.2 & $1 \times 10^{4}$ & 350 & 2 & 8.0 \\
\hline \multirow[t]{2}{*}{30} & 3 & 25 & $3 \times 10^{4}$ & 0.8 & 0.2 & $3 \times 10^{4}$ & 400 & 2 & 15.0 \\
\hline & 5 & 25 & $3 \times 10^{4}$ & 0.8 & 0.2 & $3 \times 10^{4}$ & 450 & 2 & 25.0 \\
\hline
\end{tabular}

Table 2

HAS parameter settings for data set 2

\begin{tabular}{|c|c|c|c|c|c|c|c|c|c|}
\hline $\begin{array}{l}\text { No. of } \\
\text { departments }\end{array}$ & $\begin{array}{l}\text { No. of time } \\
\text { periods }\end{array}$ & $M$ & $Q$ & $q$ & $\alpha_{1}$ & $\alpha_{2}$ & $S$ & $R$ & $\begin{array}{l}\text { Running time } \\
\text { (min) }\end{array}$ \\
\hline \multirow[t]{2}{*}{6} & 5 & 5 & $3 \times 10^{5}$ & 0.9 & 0.1 & $3 \times 10^{5}$ & 100 & 3 & 0.5 \\
\hline & 10 & 10 & $3 \times 10^{5}$ & 0.9 & 0.1 & $3 \times 10^{5}$ & 150 & 3 & 2.0 \\
\hline \multirow[t]{2}{*}{15} & 5 & 15 & $3 \times 10^{6}$ & 0.9 & 0.1 & $3 \times 10^{6}$ & 300 & 3 & 6.0 \\
\hline & 10 & 20 & $3 \times 10^{6}$ & 0.8 & 0.2 & $3 \times 10^{6}$ & 350 & 2 & 15.0 \\
\hline \multirow[t]{2}{*}{30} & 5 & 25 & $2 \times 10^{6}$ & 0.8 & 0.2 & $2 \times 10^{6}$ & 400 & 2 & 25.0 \\
\hline & 10 & 30 & $4 \times 10^{6}$ & 0.8 & 0.2 & $4 \times 10^{6}$ & 450 & 2 & 45.0 \\
\hline
\end{tabular}

and 31 problems, respectively. In other words, HAS III obtained the best solution for all the problems except problem 20, where it was within $0.2 \%$ above the best solution found. Therefore, HAS III clearly outperformed the other heuristics, and HAS II and TS were comparable with respect to solution quality for this data set.

Kaku and Mazzola [13] gave the average computation times for their TS heuristic. The average computation time for the TS heuristic for the larger size problems (problems 29-32) was approximately $2 \mathrm{~h}$ and $47 \mathrm{~min}$ on a Pentium $200 \mathrm{MHz}$ PC. For the HAS heuristics, the average computation time was $20 \mathrm{~min}$ for each run ( 3 runs) on a Pentium IV $2.4 \mathrm{GHz}$ PC.

In Table 4, out of the 48 problems the SA and GADP heuristics obtained the best solution for 2 and 13 problems, respectively. However, the HAS I, II, and III obtained the best solution for 19, 30, and 36 problems, respectively. Therefore, HAS III outperformed all of the heuristics, and HAS II and I were the second and third best heuristics with respect to solution quality for this data set. Balakrishnan et al. [17] solved the 30 department problems with 10 periods in approximately $16.7 \mathrm{~min}$ on DEC Alpha machines. 
Table 3

Solution results for data set 1

\begin{tabular}{|c|c|c|c|c|c|c|c|c|c|c|}
\hline $\mathrm{Pb} \#$ & $N$ & $T$ & $\mathrm{CP}$ & $\mathrm{TS}$ & Best found & HAS I & HAS II & HAS III & Best HAS & $\%$ Deviation \\
\hline P01 & 6 & 3 & $267 *$ & $267 *$ & 267 & 267 & 267 & 267 & 267 & 0 \\
\hline P02 & & & $260 *$ & $260 *$ & 260 & 260 & 260 & 260 & 260 & 0 \\
\hline P03 & & & $363^{*}$ & $363^{*}$ & 363 & 363 & 363 & 363 & 363 & 0 \\
\hline P04 & & & $299 *$ & $299 *$ & 299 & 299 & 299 & 299 & 299 & 0 \\
\hline P05 & & 5 & $442 *$ & $442 *$ & 442 & 442 & 442 & 442 & 442 & 0 \\
\hline P06 & & & 589 & 586 & 586 & 586 & 586 & 586 & 586 & 0 \\
\hline P07 & & & $424 *$ & $424 *$ & 424 & 424 & 424 & 424 & 424 & 0 \\
\hline P08 & & & $428 *$ & $428^{*}$ & 428 & 428 & 428 & 428 & 428 & 0 \\
\hline P09 & 12 & 3 & 1624 & 1624 & 1624 & 1624 & 1624 & 1624 & 1624 & 0 \\
\hline P10 & & & 1973 & 1973 & 1973 & 1973 & 1973 & 1973 & 1973 & 0 \\
\hline P11 & & & 1661 & 1661 & 1661 & 1661 & 1661 & 1661 & 1661 & 0 \\
\hline P12 & & & 2097 & 2097 & 2097 & 2097 & 2097 & 2097 & 2097 & 0 \\
\hline P13 & & 5 & 2930 & 2930 & 2930 & 2930 & 2930 & 2930 & 2930 & 0 \\
\hline P14 & & & 3726 & 3701 & 3701 & 3701 & 3701 & 3701 & 3701 & 0 \\
\hline P15 & & & 2756 & 2756 & 2756 & 2756 & 2756 & 2756 & 2756 & 0 \\
\hline P16 & & & 3364 & 3364 & 3364 & 3364 & 3364 & 3364 & 3364 & 0 \\
\hline P17 & 20 & 3 & 2763 & 2758 & 2758 & 2758 & 2758 & 2758 & 2758 & 0 \\
\hline P18 & & & 5318 & 5318 & 5318 & 5318 & 5318 & 5318 & 5318 & 0 \\
\hline P19 & & & 3048 & 3056 & 3048 & 3098 & 3034 & 3034 & 3034 & -0.5 \\
\hline P20 & & & 5873 & 5903 & 5873 & 5897 & 5914 & 5881 & 5881 & 0.2 \\
\hline P21 & & 5 & 4581 & 4605 & 4581 & 4575 & 4629 & 4575 & 4575 & -0.1 \\
\hline $\mathrm{P} 22$ & & & 9825 & 9746 & 9746 & 9724 & 9724 & 9724 & 9724 & -0.2 \\
\hline $\mathrm{P} 23$ & & & 4654 & 4654 & 4654 & 4667 & 4654 & 4654 & 4654 & 0 \\
\hline $\mathrm{P} 24$ & & & 8985 & 8979 & 8979 & 8979 & 8979 & 8979 & 8979 & 0 \\
\hline $\mathrm{P} 25$ & 30 & 3 & 7163 & 7130 & 7130 & 7133 & 7130 & 7130 & 7130 & 0 \\
\hline P26 & & & 14,583 & 14,478 & 14,478 & 14,478 & 14,524 & 14,478 & 14,478 & 0 \\
\hline P27 & & & 8066 & 8115 & 8066 & 8138 & 8128 & 8066 & 8066 & 0 \\
\hline P28 & & & 14,940 & 14,925 & 14,925 & 14,934 & 14,947 & 14,925 & 14,925 & 0 \\
\hline P29 & & 5 & 13,719 & 13,606 & 13,606 & 13,908 & 13,606 & 13,374 & 13,374 & -1.7 \\
\hline P30 & & & 26,027 & 25,583 & 25,583 & 25,640 & 25,747 & 25,521 & 25,521 & -0.2 \\
\hline P31 & & & 12,351 & 12,163 & 12,163 & 12,193 & 12,163 & 12,163 & 12,163 & 0 \\
\hline P32 & & & 24,409 & 24,200 & 24,200 & 24,354 & 24,200 & 24,200 & 24,200 & 0 \\
\hline
\end{tabular}

\section{Conclusion}

In this paper, the HAS for the QAP (HAS-QAP) was modified to solve the DFLP, since HASs were never used to solve this computationally intractable problem. The first heuristic, called HAS I, is a direct application of the HAS-QAP heuristic for the DFLP. However, this heuristic uses a random descent pairwise exchange heuristic to improve the set of layout plans after performing $R$ pairwise exchanges using the pheromone trail matrix. The second heuristic, called HAS II, is exactly like HAS I, except that a SA heuristic is used as the local search heuristic, instead of the random descent pairwise exchange heuristic. The third heuristic, called HAS III, is exactly like HAS I, except that the random descent pairwise exchange heuristic has a look-ahead/look-back strategy. The proposed HAS heuristics performed well for two data sets taken from the literature. 
Table 4

Solution results for data set 2

\begin{tabular}{|c|c|c|c|c|c|c|c|c|c|c|}
\hline $\mathrm{Pb} \#$ & $N$ & $T$ & SA & GADP & Best found & HAS I & HAS II & HAS III & Best found & $\%$ Deviation \\
\hline P01 & 6 & 5 & 107,249 & 106,419 & 106,419 & 106,419 & 106,419 & 106,419 & 106,419 & 0 \\
\hline P02 & & & 105,710 & 104,834 & 104,834 & 104,834 & 104,834 & 104,834 & 104,834 & 0 \\
\hline P03 & & & 104,800 & 104,320 & 104,320 & 104,320 & 104,320 & 104,320 & 104,320 & 0 \\
\hline P04 & & & 106,515 & 106,515 & 106,515 & 106,399 & 106,399 & 106,399 & 106,399 & -0.11 \\
\hline P05 & & & 106,282 & 105,628 & 105,628 & 105,628 & 105,628 & 105,628 & 105,628 & 0 \\
\hline P06 & & & 103,985 & 104,053 & 103,985 & 103,985 & 103,985 & 103,985 & 103,985 & 0 \\
\hline P07 & & & 106,447 & 106,439 & 106,439 & 106,439 & 106,439 & 106,439 & 106,439 & 0 \\
\hline P08 & & & 103,771 & 103,771 & 103,771 & 103,771 & 103,771 & 103,771 & 103,771 & 0 \\
\hline P09 & & 10 & 215,200 & 214,313 & 214,313 & 214,313 & 214,313 & 214,313 & 214,313 & 0 \\
\hline P10 & & & 214,713 & 212,134 & 212,134 & 212,134 & 212,134 & 212,134 & 212,134 & 0 \\
\hline P11 & & & 208,351 & 207,987 & 207,987 & 207,987 & 207,987 & 207,987 & 207,987 & 0 \\
\hline P12 & & & 213,331 & 212,741 & 212,741 & 212,530 & 212,530 & 212,530 & 212,530 & -0.10 \\
\hline P13 & & & 213,812 & 210,944 & 210,944 & 210,906 & 210,906 & 210,906 & 210,906 & -0.02 \\
\hline P14 & & & 211,213 & 210,000 & 210,000 & 209,932 & 209,932 & 209,932 & 209,932 & -0.03 \\
\hline P15 & & & 215,630 & 215,452 & 215,452 & 214,252 & 214,252 & 214,252 & 214,252 & -0.56 \\
\hline P16 & & & 214,513 & 212,588 & 212,588 & 212,588 & 212,588 & 212,588 & 212,588 & 0 \\
\hline P17 & 15 & 5 & 501,447 & 484,090 & 484,090 & 481,511 & 481,395 & 480,453 & 480,453 & -0.76 \\
\hline P18 & & & 506,236 & 485,352 & 485,352 & 484,761 & 484,761 & 484,879 & 484,761 & -0.12 \\
\hline P19 & & & 512,886 & 489,898 & 489,898 & 490,899 & 488,748 & 490,398 & 488,748 & -0.24 \\
\hline P20 & & & 504,956 & 484,625 & 484,625 & 485,561 & 485,658 & 484,446 & 484,446 & -0.04 \\
\hline $\mathrm{P} 21$ & & & 509,636 & 489,885 & 489,885 & 489,012 & 487,722 & 489,206 & 487,722 & -0.44 \\
\hline $\mathrm{P} 22$ & & & 508,215 & 488,640 & 488,640 & 487,417 & 486,685 & 486,965 & 486,685 & -0.40 \\
\hline $\mathrm{P} 23$ & & & 508,848 & 489,378 & 489,378 & 486,853 & 486,853 & 486,853 & 486,853 & -0.52 \\
\hline $\mathrm{P} 24$ & & & 512,320 & 500,779 & 500,779 & 493,963 & 492,074 & 491,016 & 491,016 & -1.99 \\
\hline $\mathrm{P} 25$ & & 10 & $1,017,741$ & 987,887 & 987,887 & 982,208 & 980,351 & 980,351 & 980,351 & -0.77 \\
\hline P26 & & & $1,016,567$ & 980,638 & 980,638 & 981,802 & 978,271 & 980,197 & 978,271 & -0.24 \\
\hline $\mathrm{P} 27$ & & & $1,021,075$ & 985,886 & 985,886 & $\mathbf{9 7 8 , 0 2 7}$ & 985,056 & 984,065 & 978,027 & -0.80 \\
\hline P28 & & & $1,007,713$ & 976,025 & 976,025 & 978,987 & 974,694 & 975,854 & 974,694 & -0.14 \\
\hline P29 & & & $1,010,822$ & 982,778 & 982,778 & 980,611 & 980,548 & 979,196 & 979,196 & -0.37 \\
\hline P30 & & & $1,007,210$ & 973,912 & 973,912 & 973,508 & 971,827 & $\mathbf{9 7 1 , 5 4 8}$ & 971,548 & -0.24 \\
\hline P31 & & & $1,013,315$ & 982,872 & 982,872 & 985,659 & 982,951 & 980,752 & 980,752 & -0.22 \\
\hline P32 & & & $1,019,092$ & 987,789 & 987,789 & 987,986 & 986,087 & 985,707 & 985,707 & -0.21 \\
\hline P33 & 30 & 5 & 604,408 & 578,689 & 578,689 & 578,854 & $\mathbf{5 7 6 , 8 8 6}$ & 580,240 & 576,886 & -0.31 \\
\hline P34 & & & 604,370 & 572,232 & 572,232 & 570,349 & 571,528 & 570,349 & 570,349 & -0.33 \\
\hline P35 & & & 603,867 & 578,527 & 578,527 & 578,152 & $\mathbf{5 7 6 , 0 5 3}$ & 578,176 & 576,053 & -0.43 \\
\hline P36 & & & 596,901 & 572,057 & 572,057 & 569,694 & 572,005 & 566,777 & 566,777 & -0.93 \\
\hline P37 & & & 591,988 & 559,777 & 559,777 & 560,433 & 558,353 & 558,353 & 558,353 & -0.26 \\
\hline P38 & & & 599,862 & 566,792 & 566,792 & 569,725 & 570,567 & 566,792 & 566,792 & 0 \\
\hline P39 & & & 600,670 & 567,873 & 567,873 & 570,899 & 567,190 & 567,131 & 567,131 & -0.13 \\
\hline P40 & & & 610,474 & 575,720 & 575,720 & 576,980 & 575,998 & 575,280 & 575,280 & -0.08 \\
\hline P41 & & 10 & $1,223,124$ & $1,169,474$ & $1,169,474$ & $1,166,164$ & $1,169,688$ & $1,166,164$ & $1,166,164$ & -0.28 \\
\hline P42 & & & $1,231,151$ & $1,168,878$ & $1,168,878$ & $1,169,407$ & $1,177,398$ & $1,168,878$ & $1,168,878$ & 0 \\
\hline P43 & & & $1,230,520$ & $1,166,366$ & $1,166,366$ & $1,166,916$ & $1,166,366$ & $1,166,720$ & $1,166,366$ & 0 \\
\hline P44 & & & $1,200,613$ & $1,154,192$ & $1,154,192$ & $1,190,140$ & $1,148,202$ & $1,148,202$ & $1,148,202$ & -0.52 \\
\hline P45 & & & $1,210,892$ & $1,133,561$ & $1,133,561$ & $1,128,855$ & $1,142,776$ & $1,133,664$ & $1,128,855$ & -0.42 \\
\hline
\end{tabular}


Table 4 (continued)

\begin{tabular}{|c|c|c|c|c|c|c|c|c|c|c|}
\hline $\mathrm{Pb} \#$ & $N$ & $T$ & SA & GADP & Best found & HAS I & HAS II & HAS III & Best found & $\%$ Deviation \\
\hline P46 & & & $1,221,356$ & $1,145,000$ & $1,145,000$ & $1,153,982$ & $1,141,344$ & $1,149,040$ & $1,141,344$ & -0.32 \\
\hline P47 & & & $1,212,273$ & $1,145,927$ & $1,145,927$ & $1,152,805$ & $1,151,735$ & $1,140,773$ & $1,140,773$ & -0.45 \\
\hline P48 & & & $1,231,408$ & $1,168,657$ & $1,168,657$ & $1,172,787$ & $1,170,349$ & $1,166,157$ & $1,166,157$ & -0.21 \\
\hline
\end{tabular}

\section{Acknowledgements}

The authors would like to thank Professors Jaydeep Balakrishnan and Thomas Lacksonen for providing the data sets. Also, the authors would like to thank the referees for their valuable comments, which greatly improved the paper.

\section{References}

[1] Tompkins JA, White JA, Bozer YA, Frazelle EH, Tanchoco JMA, Trevino J. Facilities planning. New York: Wiley; 1996. p. $137-285$.

[2] Meller RD, Gau KY. The facility layout problem: recent and emerging trends and perspectives. Journal of Manufacturing Systems 1996;15(5):351-66.

[3] Shore RH, Tompkins JA. Flexible facilities design. AIIE Transactions 1980;12(2):200-5.

[4] Rosenblatt MJ. The dynamics of plant layout. Management Sciences 1986;32(1):76-86.

[5] Ballou R. Dynamic warehouse location analysis. Journal of Marketing Research 1968;5(3):271-6.

[6] Lacksonen TA, Enscore EE. Quadratic assignment algorithms for the dynamic layout problem. International Journal of Production Research 1993;31(3):503-17.

[7] Pardalos PH, Crouse JV. A parallel algorithm for the QAP. Proceedings of the 1989 supercomputer conference. New York: ACM Press; 1989. p. 351-60.

[8] Burkard RE, Bonniger T. A heuristic for quadratic Boolean problems with application to quadratic assignment problem. European Journal of Operational Research 1983;13:374-86.

[9] Gomory RE, Hu TC. Multi-terminal network flows. SIAM Journal 1961;9:551-70.

[10] Armour GC, Buffa ES. A heuristic algorithm and simulation approach to relative location of facilities. Management Sciences 1963;9:294-309.

[11] Urban TL. A heuristic for the dynamic facility layout problem. IIE Transactions 1993;25(4):57-63.

[12] Conway DG, Venkataramanan MA. Genetic search and the dynamic facility layout problem. Computers \& Operations Research 1994;21(8):955-60.

[13] Kaku BK, Mazzola JB. A tabu search heuristic for the dynamic plant layout problem. INFORMS Journal on Computing 1997;9(4):374-84.

[14] Balakrishnan J, Cheng CH. Genetic search and the dynamic layout problem. Computers \& Operations Research 2000;27(6):587-93.

[15] Balakrishnan J, Cheng CH, Conway G. An improved pair-wise exchange heuristic for the dynamic plant layout problem. International Journal of Production Research 2000;38(13):3067-77.

[16] Baykasoglu A, Gindy NNZ. A simulated annealing algorithm for dynamic facility layout problem. Computers \& Operations Research 2001;28(14):1403-26.

[17] Balakrishnan J, Cheng CH, Conway DG, Lau CM. A hybrid genetic algorithm for the dynamic plant layout problem. International Journal of Production Economics 2003;86:107-20.

[18] Balakrishnan J, Cheng CH. Dynamic layout algorithms: a state-of-the-art survey. Omega, International Journal of Management Sciences 1998;26:507-21.

[19] Dorigo M, Gambardella LM. Ant colonies for the traveling salesman problem. BioSystems 1997;43:73-81.

[20] Gambardella LM, Taillard ED, Dorigo M. Ant colonies for the quadratic assignment problem. Journal of Operational Research Society 1999;50:167-76. 
[21] Colorni A, Dorigo M, Maniezzo V. Distributed optimization by ants colonies. In: Varela F, Bourgine P, editors. First European conference on artificial life, 1991. p. 134-42.

[22] Maniezzo V, Colorni A. The ant system applied to the quadratic assignment problem. IEEE Transactions on Knowledge and Data Engineering 1999;11:769-78.

[23] Stutzle T, Dorigo M, ACO algorithms for the quadratic assignment problem. In: Corne D, Dorigo M, Glover F, editors. New ideas in optimization. New York: McGraw-Hill; 1999. p. 33-50.

[24] Kirkpatrick S, Gelatt CD, Vecchi MP. Optimization by simulated annealing. Science 1983;220(4598):671-80.

[25] Wilhelm MR, Ward TL. Solving quadratic assignment problems by simulated annealing. IIE Transactions 1987;19(1): 107-19.

[26] Heragu SS, Alfa AS. Experimental analysis of simulated annealing based algorithms for the layout problem. European Journal of Operational Research 1992;57:190-202.

[27] Baykasoglu A, Gindy NNZ. Erratum to a simulated annealing algorithm for dynamic facility layout problem [Computers \& Operations Research 28 (2001) 1367-460]. Computers \& Operations Research 2004;31(2):313-5. 